\title{
It's Time for a Strategic Approach to Observation Care
}

\author{
Amber K. Sabbatini, MD, MPH ${ }^{1}$, Renee Y. Hsia, MD, MSc ${ }^{2,3^{*}}$
}

${ }^{1}$ Division of Emergency Medicine, University of Washington, Seattle, Washington; ${ }^{2}$ Department of Emergency Medicine, University of California San Francisco, San Francisco, California; ${ }^{3}$ Philip R. Lee Institute for Health Policy Studies, University of California San Francisco, San Francisco, California.

After patients have experienced an illness requiring a hospital stay, they are increasingly finding that despite having received treatment in a hospital bed, they were never actually admitted - at least not from the perspective of their insurers. Instead, these patients were kept under observation, an outpatient designation that allows a hospital to bill for observation services without formally admitting a patient.

Recent studies have recorded significant increases in hospitals' use of observation stays among the Medicare population, ${ }^{1-3}$ raising concerns about the financial ramifications for patients. Under observation, patients are potentially responsible for a greater share of the cost and bear the financial consequences of inappropriate observation stays. Currently, around $6 \%$ of Medicare patients hospitalized as outpatients spend more than 48 hours (or two midnights) in observation, sometimes much longer, exposing them to significant out-of-pocket costs. ${ }^{3}$ In addition, liberal use of observation can lead to increased hospital stays, for example among lower-severity emergency department (ED) patients who could have been safely discharged but were instead kept for a costly observation stay. ${ }^{4}$ At the same time, hospitals do not necessarily benefit from this cost shifting; in fact, hospital margin is worse for patients under Medicare observation care. ${ }^{5}$ Yet hospitals are obligated to be compliant with CMS observation regulations and may try to avoid the consequences (eg, audits, non-payment) for inpatient stays that are deemed inappropriate by CMS.

While the nuances of how CMS finances observation stays have made the practice controversial, the use of observation care in other payer groups that may not have the same reimbursement policies, and its impact on patients, have not been well studied. In this issue of the Journal of Hospital Medicine, Nuckols et al. ${ }^{6}$ begins to address this gap by carefully exploring trends in observation stays in a multipayer data set.

The authors use data for four states (Georgia, Nebraska, South Carolina, and Tennessee) from the Healthcare Cost and Utilization Project (Agency for Healthcare Quality and Research) and the American Community Survey (US Census Bureau) to calculate population based rates of ED visits, observation stays, and inpatient admissions. To date, this

*Address for correspondence and reprint requests: Renee Y. Hsia, MD, MSc, Department of Emergency Medicine, University of California San Francisco, 1001 Potrero Ave, 1E21, San Francisco General Hospital, San Francisco, CA 94110; Telephone: 415-206-4612; Fax: 415-206-5818; E-mail: renee.hsia@ ucsf.edu

Received: March 7, 2017; Revised: May 9, 2017; Accepted: May 10, 2017

2017 Society of Hospital Medicine DOI 10.12788/jhm.2760 is the first study to examine and compare the use of observation stays in an all-payer data set. Similar to prior work that examined the Medicare population, the authors find increased rates of treat-and-release ED visits and observation stays over time with a corresponding decline in inpatient admissions. As this study clearly shows, observation stays are comprising a greater fraction of the total hospital care delivered to patients with acute illnesses.

In many ways, the findings of Nuckols et al. ${ }^{6}$ raise more questions than they answer. For example, does the rise in observation stays represent a fundamental shift in how hospitals deliver care, an alternative to costly inpatient admissions? Are changing payer incentives driving hospitals to be more prudent in their inpatient admission practices, or are similar services simply being delivered under a new billing designation? And, most important, does this shift have any repercussions for the quality and safety of patient care?

Ultimately, the answer to these questions is, "It depends." As the authors mention, most US hospitals admit observation patients to general medical wards, where they receive care at the admitting provider's discretion instead of utilizing specific care pathways or observation protocols. ${ }^{7}$ In some of these hospitals, there may be little to no difference in how the observation patient is treated compared with a similar patient who is hospitalized as an inpatient.

However, a minority of hospitals has been more strategic in their delivery of observation care and have developed observation units. While observation units vary in design, common features include a dedicated location in the hospital with dedicated staff, reliance on clear inclusion-exclusion criteria for admission to the unit, and the use of rapid diagnostic or treatment protocols for a limited number of conditions. About half of these observation units are ED-based, reducing transitions of care between services. Protocol-driven observation units have the potential to prevent unnecessary inpatient admissions, standardize evidence-based practice, and reduce practice variation and resource use, apparently without increasing adverse events. ${ }^{8}$ In addition, they may also lead to better experiences of care for many patients compared with inpatient admissions.

Medicare's own policy on observation hospital care succinctly describes ED observation units: "Observation services are commonly ordered for patients who present to the emergency department and who then require a significant period of treatment in order to make a decision concerning their admission or discharge... usually in less than 24 hours." Due to regulatory changes and auditing pressure, observation care has expanded beyond this definition in length of 
stay, scope, and practice such that much of observation care now occurs on general hospital wards. Ideally, observation policy must be realigned with its original intent and investment made in ED observation units.

The shifting landscape of hospital-based care as described by Nuckols et al. ${ }^{6}$ highlights the need for a more strategic approach to the delivery of acute care. Unfortunately, to date, there has been a lack of attention among policymakers towards promoting a system of emergent and urgent care that is coordinated and efficient. Observation stays are one major area for which innovations in the acute care delivery system may result in meaningful improvement in patient outcomes and greater value for the healthcare system. Incentivizing a system of high-value observation care, such as promoting the use of observation units that employ evidence-based practices, should be a key priority when considering approaches to reducing the cost of hospital-based and other acute care.

One strategy is to better define and possibly expand the cohort of patients likely to benefit from care in an observation unit. Hospitals with significant experience using observation units treat not only common observation conditions like chest pain, asthma, or cellulitis, but also higher-risk inpatient conditions like syncope and diabetic ketoacidosis using rapid diagnostic and treatment protocols.

Identifying high-value observation care also will require developing patient outcome measures specific for observation stays. Observation-specific quality measures will allow a comparison of hospitals that use different care pathways for observation patients or treat certain populations of patients in observation units. This necessitates looking beyond resource use (costs and length of stay), which most studies on observation units have focused on, and examining a broader range of patient outcomes like time to symptomatic resolution, quality of life, or return to productivity after an acute illness.

Finally, observation care is also a good target for payment redesign. For example, incentive payments could be provided to hospitals that choose to develop observation units, employ observation units that utilize best known practices for observation care (such as protocols and clearly defined patient cohorts), or deliver particularly good acute care outcomes for patients with observation-amenable conditions. On the consumer side, value-based contracting could be used to shunt patients with acute conditions that require evaluation in an urgent care center or ED to hospitals that use observation units.

While the declines in inpatient admission and increases in treat-and-release ED patients have been well-documented over time, perhaps the biggest contribution of this study from Nuckols et al. ${ }^{6}$ lies in its identification of the changes in observation care, which have been increasing in all payer groups. Our opportunity now is to shape whether these shifts toward observation care deliver greater value for patients.

\section{Acknowledgment}

The authors thank Joanna Guo, BA, for her editorial and technical assistance.

Disclosure: Nothing to report.

\section{References}

1. Feng Z, Wright B, Mor V. Sharp rise in Medicare enrollees being held in hospitals for observation raises concerns about causes and consequences. Health Aff (Millwood). 2012;31(6):1251-1259.

2. Zuckerman RB, Sheingold SH, Orav EJ, Ruhter J, Epstein AM. Readmissions, observation, and the Hospital Readmissions Reduction Program. N Engl J Med. 2016;374(16):1543-1551

3. Office of Inspector General. Vulnerabilities Remain Under Medicare's 2-Midnight Hospital Policy. US Department of Health \& Human Services. Published 2016. https://oig.hhs.gov/oei/reports/oei-02-15-00020.pdf. Accessed April 25, 2017.

4. Blecker S, Gavin NP, Park H, Ladapo JA, Katz SD. Observation units as substitutes for hospitalization or home discharge. Ann Emerg Med. 2016;67(6):706-713.e702.

5. Medicare Payment Advisory Commission. Report to the Congress: Medicare Payment Policy. Published 2015. http://medpac.gov/docs/default-source/reports/ mar2015_entirereport_revised.pdf?sfvrsn=0). Accessed April 25, 2017.

6. Nuckols TN, Fingar KR, Barrett M, Steiner C, Stocks C, Owens PL. The shifting landscape in utilization of inpatient, observation, and emergency department services across payers. J Hosp Med. 2017;12(6):444-446.

7. Ross MA, Hockenberry JM, Mutter R, Barrett M, Wheatley M, Pitts SR. Protocol-driven emergency department observation units offer savings, shorter stays, and reduced admissions. Health Aff (Millwood). 2013;32(12):2149-2156.

8. Ross MA, Aurora T, Graff L, et al. State of the art: emergency department observation units. Crit Pathw Cardiol. 2012;11(3):128-138. 